\title{
Bioconductance Measurement
}

National Cancer Institute

\section{Source}

National Cancer Institute. Bioconductance Measurement. NCI Thesaurus. Code C116550.

A test that uses electrical current conductance as measured through skin to help diagnose disease, most commonly lung tumors. 